\title{
New Results for Set-Valued Mappings on Ordered Metric Spaces
}

\author{
F. Sabetghadam, ${ }^{1}$ H. P. Masiha, ${ }^{1}$ and H. Aydi $\mathbb{D}^{2,3,4}$ \\ ${ }^{1}$ Faculty of Mathematics, K. N. Toosi University of Technology, P.O. Box 16315-1618, Tehran, Iran \\ ${ }^{2}$ Nonlinear Analysis Research Group, Ton Duc Thang University, Ho Chi Minh City, Vietnam \\ ${ }^{3}$ Faculty of Mathematics and Statistics, Ton Duc Thang University, Ho Chi Minh City, Vietnam \\ ${ }^{4}$ China Medical University Hospital, China Medical University, Taichung 40402, Taiwan \\ Correspondence should be addressed to H. Aydi; hassen.aydi@tdtu.edu.vn
}

Received 6 February 2020; Accepted 22 August 2020; Published 24 September 2020

Academic Editor: Lars E. Persson

Copyright $\odot 2020$ F. Sabetghadam et al. This is an open access article distributed under the Creative Commons Attribution License, which permits unrestricted use, distribution, and reproduction in any medium, provided the original work is properly cited.

We establish some new fixed point results for order-closed multivalued mappings in complete metric spaces endowed with a partial order.

\section{Introduction}

In 1976, an order relation was defined in metric spaces [1]. Later, many researchers proved various fixed point results in this setting (see [2-11]), while the authors in $[4,5,9]$ considered coupled questions for so-called monotone conditions. Zhang [12] proved some (coupled) fixed point theorems for multivalued mappings with monotone conditions in metric spaces with a partial order. Since then, Agarwal and Khamsi [13] extended Caristi's fixed point to vector-valued metric spaces. Also, Chung [14] considered nonlinear contraction mappings. Nadler [15] defined multivalued contractions, and Assad and Kirk [16] proved fixed point theorems for setvalued mappings (see also the related works [17-22]).

In this paper, we present some fixed point results in ordered metric spaces for order-closed multivalued operators. First, we need some facts.

Lemma 1. [23] Let $(\mathscr{E}, d)$ be a metric space and $\phi: \mathscr{E} \longrightarrow$ $(-\infty,+\infty)$ be a functional. Consider $\gamma:[0,+\infty) \longrightarrow[0,+\infty)$ a nondecreasing, continuous, and subadditive function so that $\gamma^{-1}(\{0\})=\{0\}$. Take the relation “ $\leqslant$ ” on $\mathscr{E}$ given as

$$
u \preccurlyeq v \text { iff } \gamma(d(u, v)) \leq \phi(u)-\phi(v) \text {, for all } u, v \in \mathscr{E}
$$

Then, “ $\preccurlyeq$ " is a partial order relation on $\mathscr{E}$. Apparently, $u \preccurlyeq v$ then $\phi(u) \geq \phi(v)$.
Here, we state some definitions. Let $\mathscr{E}$ be a topological space. Denote by $\mathcal{N}(\mathscr{E})$ the family of nonempty subsets of $\mathscr{E}$. Let $\preccurlyeq$ be a partial order on $\mathscr{E}$.

Definition 2. [24] Given two nonempty subsets $U$ and $V$ of $\mathscr{E}$.

(r1) If for each $a \in U$, there is $b \in V$ so that $a \preccurlyeq b$, then $U \prec_{1} V$

(r2) If for each $b \in V$, there is $a \in U$ so that $a \leqslant b$, then $U \prec_{2} V$

(r3) If $U \prec_{1} V$ and $U \prec_{2} V$, then $U \prec V$.

Note that $\prec_{1}$ and $\prec_{2}$ are different relations between $U$ and $V$ (see Remark 114 of [24]). Also, $\prec_{1}, \prec_{2}$, and $\prec$ are not partial orders on $\mathcal{N}(\mathscr{E})$ (see Remark 2 of [24]).

Definition 3. If $\left\{u_{n}\right\} \subset \mathscr{E}$ satisfies $u_{1} \preccurlyeq u_{2} \preccurlyeq \cdots \preccurlyeq u_{n} \preccurlyeq \cdots$ or $u_{1} \geqslant$ $u_{2} \geqslant \cdots \geqslant u_{n} \geqslant \cdots$, then $\left\{u_{n}\right\}$ is called a monotone sequence.

Definition 4. A multivalued operator $G: \mathscr{E} \longrightarrow \mathcal{N}(\mathscr{E})$ is said to be order-closed if, for monotone sequences $\left\{u_{n}\right\}$ and $\left\{v_{n}\right\} \subset \mathscr{E}$, we have $u_{n} \longrightarrow u_{0}, v_{n} \longrightarrow v_{0}$, and $v_{n} \in G u_{n}$ imply $v_{0} \in G u_{0}$.

Definition 5. A function $g: \mathscr{E} \longrightarrow(-\infty,+\infty)$ is said to be order upper (lower) semicontinuous, if, for a monotone sequence $\left\{u_{n}\right\} \subset \mathscr{E}$ with $u_{0} \in \mathscr{E}$, we have 


$$
u_{n} \longrightarrow u_{0} \Rightarrow \varlimsup_{n \infty} g\left(u_{n}\right) \leq g\left(u_{0}\right),\left(g\left(u_{0}\right) \leq \lim _{\overline{n \infty}} g\left(u_{n}\right)\right)
$$

Note that an upper (lower) semicontinuous function is an order upper (lower) semicontinuous. But the converse is not true (see Remark 3 of [24]).

\section{Main Results}

Let $(\mathscr{E}, d)$ be a metric space. For $\phi: \mathscr{E} \longrightarrow \mathbb{R}$, define the partial order " $\leqslant$ " on $\mathscr{E}$ induced by $\phi$ and $\gamma$ in Lemma 1.

Theorem 6. Let $(\mathscr{E}, d, \preccurlyeq)$ be a complete ordered metric space and $\phi: \mathscr{E} \longrightarrow(-\infty,+\infty)$ be a bounded below function. Suppose that $G: \mathscr{E} \longrightarrow \mathcal{N}(\mathscr{E})$ is order-closed with respect to " $\leqslant$ " so that

\section{(H) for any $u \in \mathscr{E}$, there is $v \in G u$ so that $u \leqslant v$}

Then, there is a monotone sequence $\left\{u_{n}\right\}_{n=0}^{\infty} \subset \mathscr{E}$, so that $u_{n+1} \in G u_{n}$ for $n=0,1,2, \cdots$, and $\left\{u_{n}\right\}$ converges to $u^{*}$, which is a fixed point of $G$. If in addition, $\phi$ is order lower semicontinuous on $\mathscr{E}$, then $u_{n} \leqslant u^{*}$ for each $n$.

Proof. By the condition $(H)$, take $u_{0} \in \mathscr{E}$. From $(H)$, there is $u_{1} \in G u_{0}$ so that $u_{0} \leqslant u_{1}$. Again, from $(H)$, there is $u_{2} \in G u_{1}$ with $u_{1} \leqslant u_{2}$. Continuing this procedure, we have an increasing sequence $\left\{u_{n}\right\}$ so that $u_{n+1} \in G u_{n}$. Therefore,

$$
\phi\left(u_{n-1}\right) \geq \phi\left(u_{n}\right) \text { for all } n .
$$

That is, the real sequence $\left\{\phi\left(u_{n}\right)\right\}$ is decreasing, so it is a convergent sequence because $\phi$ is bounded from below on $\mathscr{E}$. that

Using Remark 3 of [25] and Remark 2 of [26], we find

$$
\lim _{\ell \rightarrow 0^{+}} \frac{\gamma(\ell)}{\ell}=\sup \left\{\frac{\gamma(\ell)}{\ell}: \ell>0\right\}
$$

provided that $\gamma$ is subadditive. Thus,

$$
\liminf _{\ell \rightarrow 0^{+}} \frac{\gamma(\ell)}{\ell}>0
$$

By (7), there are $\delta>0$ and $c>0$ so that

$$
\gamma(\ell) \geq c \ell, \text { for all } \ell \in[0, \delta] .
$$

Since $\gamma$ is nondecreasing, we have $\gamma(\ell) \geq \gamma(\delta)$ for each $\ell \in[\delta,+\infty)$.

Let $0<\varepsilon<\gamma(\delta)$. Then

$$
\gamma(\ell)>\varepsilon \text {, for each } \ell \in[\delta,+\infty),
$$

i.e.,

$$
\text { if } \gamma(\ell) \leq \varepsilon \text {, then } \ell \in[0, \delta] \text {. }
$$

Therefore, we have

$$
\{\ell \geq 0: \gamma(\ell) \leq \varepsilon\} \subset[0, \delta],
$$

which together with (8) implies that

$$
\gamma(\ell) \geq c \ell, \text { for all } \ell \in\{\ell \geq 0: \gamma(\ell) \leq \varepsilon\} .
$$

Note that $\left\{\phi\left(u_{n}\right)\right\}$ is convergent; then, there is $n_{0}$ so that for all $m \geq n \geq n_{0}$,

$$
\gamma\left(d\left(u_{n}, u_{m}\right)\right) \leq \phi\left(u_{n}\right)-\phi\left(u_{m}\right)<\varepsilon .
$$

Moreover, by (12), we get

$c d\left(u_{n}, u_{m}\right) \leq \gamma\left(d\left(u_{n}, u_{m}\right)\right) \leq \phi\left(u_{n}\right)-\phi\left(u_{m}\right)$, for all $m \geq n \geq n_{0}$.

The convergence of $\left\{\phi\left(u_{n}\right)\right\}$ implies that $\left\{u_{n}\right\}$ is a Cauchy sequence. By the completeness of $\mathscr{E}$, there is $u^{*} \in \mathscr{E}$ so that $u_{n} \longrightarrow u^{*}$ as $n \longrightarrow \infty$. Since $G$ is order-closed, $\left\{u_{n}\right\}$ is monotone and $u_{n+1} \in G u_{n}$. Consequently,

$$
u^{*} \in G u^{*}
$$

i.e., $u^{*}$ is a fixed point of $G$.

If $\phi$ is order lower semicontinuous on $\mathscr{E}$, by definition of “ミ," then we have for each $m$

$$
\begin{aligned}
\gamma\left(d\left(u_{m}, u^{*}\right)\right) & =\lim _{n \rightarrow \infty} \gamma\left(d\left(u_{m}, u_{n}\right)\right) \leq \varlimsup_{n \infty}\left(\phi\left(u_{m}\right)-\phi\left(u_{n}\right)\right) \\
& =\phi\left(u_{m}\right)-\varliminf_{\overline{n \infty}} \phi\left(u_{n}\right) \leq \phi\left(u_{m}\right)-\phi\left(u^{*}\right) .
\end{aligned}
$$

It yields that $u_{m} \leqslant u^{*}$. The proof is completed.

The proof of the following theorem carries over in the same manner as for Theorem 6 .

Theorem 7. Let $(\mathscr{E}, d, \preccurlyeq)$ be a complete ordered metric space and $\phi: \mathscr{E} \longrightarrow(-\infty,+\infty)$ be a bounded below function. Suppose that $G: \mathscr{E} \longrightarrow \mathcal{N}(\mathscr{E})$ is order-closed with respect to "ミ." Assume that

$$
\text { (H) for each } u \in \mathscr{E} \text {, there is } v \in \text { Gu so that } v \leqslant u
$$

Then, there is a monotone sequence $\left\{u_{n}\right\}_{n=0}^{\infty} \subset \mathscr{E}, u_{n+1} \in$ $G u_{n}, n=0,1,2, \cdots$, such that $\left\{u_{n}\right\}$ converges to $u^{*}$, which is a fixed point of $G$. If, in addition, $\phi$ is order upper semicontinuous on $\mathscr{E}$, then $u^{*} \leqslant u_{n}$ for all $n$.

Theorem 8. Let $(\mathscr{E}, d, \preccurlyeq)$ be a complete ordered metric space and $\phi: \mathscr{E} \longrightarrow(-\infty,+\infty)$ be bounded below function. Suppose that $G: \mathscr{E} \longrightarrow \mathcal{N}(\mathscr{E})$ is order-closed with respect to "ミ." Assume that

(i) for each $u, v \in \mathscr{E}$ with $u \leqslant v \Rightarrow G u \prec_{1} G v$ 
(ii) there exists $u_{0} \in \mathscr{E}$ such that, $\left\{u_{0}\right\} \prec_{1} G u_{0}$.

Then, $G$ has a fixed point $u^{*}$, and there is a sequence $\left\{u_{n}\right\}$ with $u_{n-1} \preccurlyeq u_{n} \in G u_{n-1}$ for $n \geq 1$, such that $u_{n} \longrightarrow x^{*}$. Moreover, if $\phi$ is order lower semicontinuous, then $u_{n} \preccurlyeq u^{*}$ for all $n$.

Proof. Since $G u \neq \varnothing$, by (ii), we can choose $u_{1} \in G u_{0}$ so that $x_{0} \preccurlyeq u_{1}$. This implies that $G u_{0} \prec_{1} G u_{1}$, by definition of $\prec_{1}$, there is $u_{2} \preccurlyeq G u_{1}$ so that $u_{1} \preccurlyeq u_{2}$. Continuing this procedure, we can find an increasing sequence $\left\{u_{n}\right\}$ such that $u_{n} \in G$ $u_{n-1}$. The rest of the proof is similar as in Theorem 6 .

The following supports Theorem 8 .

Example 9. Let $\mathscr{E}=[0,1]$ and $d(\xi, \sigma)=|\xi-\sigma|$, for $\xi, \sigma \in \mathscr{E}$. The metric space $(\mathscr{E}, d)$ is complete. Consider the multivalued mapping $G: \mathscr{E} \longrightarrow \mathcal{N}(\mathscr{E})$ given as

$$
G(\xi)=\left(\begin{array}{ll}
{\left[\frac{\xi}{5}, \frac{\xi}{4}\right] \text { for }} & \xi \in \mathscr{E} \cap \mathbb{Q} \\
{\left[\frac{\xi}{5=3}, \frac{\xi}{2}\right] \text { for } \quad \xi \in \mathscr{E} \mathbb{Q},}
\end{array}\right.
$$

where $\mathbb{Q}$ is the set of rational numbers. Let $\gamma(s)=s(s \geq 0)$ and $\phi(\xi)=|\xi|(\xi \in \mathscr{E})$. Note that $\phi$ is bounded from below. Take the order $\preccurlyeq$ induced by $\phi$, that is, given as follows:

$$
\xi \preccurlyeq \sigma \Leftrightarrow \gamma(d(\xi, \sigma)) \leq \phi(\xi)-\phi(\sigma) .
$$

Mention that $G$ verifies the following assertions:

(a) for each $u, v \in \mathscr{E}$ with $u \leqslant v$, we have $G u \prec_{1} G v$

(b) $0 \prec_{1} G 0$

(c) $G$ is order-closed on $\mathscr{E}$.

Hence, $G$ has a fixed point on $\mathscr{E}$, which is $u^{*}=0$.

Theorem 10. Let $(\mathscr{E}, d, \preccurlyeq)$ be a complete ordered metric space and $\phi: \mathscr{E} \longrightarrow(-\infty,+\infty)$ be a bounded below function. Suppose $G: \mathscr{E} \longrightarrow \mathcal{N}(\mathscr{E})$ is order-closed with respect to "ß." If the following conditions hold:

(i) for each $u, v \in \mathscr{E}$ with $u \preccurlyeq v \Rightarrow G u{ }_{2} G v$

(ii) there exists $u_{0} \in \mathscr{E}$ such that, $G u_{0} \prec_{2}\left\{u_{0}\right\}$

then $G$ has a fixed point $u^{*}$. Also, there is a sequence $\left\{u_{n}\right\}$ with $u_{n-1} \geqslant u_{n} \in G u_{n-1}$ for any $n \geq 1$ such that $u_{n} \longrightarrow u^{*}$. Moreover, if $\phi$ is order upper semicontinuous, then $u_{n} \geqslant u^{*}$ for all $n$.

The following example illustrates Theorem 10.

Example 11. Let $\mathscr{E}=[0,1], d(\xi, \sigma)=|\xi-\sigma|, \phi(\xi)=-\xi$ for $\xi, \sigma \in \mathscr{E}$ and $\gamma(t)=t$ for each $t \geq 0$. Here, $\mathscr{E}$ is a complete metric space, and $\phi$ is a bounded below function. Consider the order $\preccurlyeq$ induced by $\phi$ :

$$
\xi \preccurlyeq \sigma \Leftrightarrow d(\xi, \sigma) \leq \phi(\xi)-\phi(\sigma) .
$$

Clearly, this partial order is the usual order on $\mathscr{E}$. Define $G: \mathscr{E} \longrightarrow \mathcal{N}(\mathscr{E})$ by $G \xi=[(\xi / 3),(\xi / 2)]$. It is obvious that $G$ satisfies the following:

(a) for each $u, v \in \mathscr{E}$ with $u \preccurlyeq v \Rightarrow G u \prec{ }_{2} G v$

(b) $G 1 \prec_{2}\{1\}$

(c) $G$ is order-closed on $\mathscr{E}$.

Hence, $G$ has a fixed point on $\mathscr{E}$, which is $u^{*}=0$.

Now, a multivalued version of Theorem 2 of [27] may be obtained. Here, the considered multivalued mapping is not necessarily continuous.

Theorem 12. Let $(\mathscr{E}, d, \preccurlyeq)$ be a complete ordered metric space and $\phi: \mathscr{E} \longrightarrow(-\infty,+\infty)$ be a continuous function bounded below. Let $G: \mathscr{E} \longrightarrow \mathcal{N}(\mathscr{E})$ be a multivalued mapping. Suppose that

(H1) for any $u \in \mathscr{E}$ there is $v \in G u$ so that $u \preccurlyeq v$

(H2) $G(u)$ is compact for each $u \in \mathscr{E}$.

Then, G has a fixed point.

Proof. Set

$$
M:=\{u \in \mathscr{E} \mid \exists v \in G u: v \geqslant u\}
$$

We claim that $M$ has a maximum element. For a directed set $I$, let $\left\{u_{i}\right\}_{i \in I}$ be a totally ordered subset in $M$. For $i, j \in I$ with $i \leq j$, the fact that $u_{i} \preccurlyeq u_{j}$ yields $\phi\left(u_{i}\right) \geq \phi\left(u_{j}\right)$. Due to the fact that $\phi$ is bounded below, $\left\{\phi\left(u_{i}\right)\right\}$ is a convergent set in $\mathbb{R}$. Consider

$$
\gamma\left(d\left(u_{i}, u_{j}\right)\right) \leq \phi\left(u_{i}\right)-\phi\left(u_{j}\right)
$$

As in proof of Theorem 6, $\left\{u_{i}\right\}$ is Cauchy in $\mathscr{E}$, which is complete, so $\left\{u_{i}\right\}$ converges to $u$ in $\mathscr{E}$. For $j \in I$,

$$
\begin{aligned}
\gamma\left(d\left(u_{j}, u\right)\right) & =\lim _{i} \gamma\left(d\left(u_{j}, u_{i}\right)\right) \leq \lim _{i}\left(\phi\left(u_{j}\right)-\phi\left(u_{i}\right)\right) \\
& =\phi\left(u_{j}\right)-\phi(u) .
\end{aligned}
$$

Hence, $u_{j} \preccurlyeq u$ for each $j \in I$. By $(H 1)$, for each $v_{j} \in G u_{j}$, there is $w_{j} \in G u$ so that $v_{j} \preccurlyeq w_{j}$. By the compactness of $G u$, there is a convergence subset $\left\{v_{j}^{\prime}\right\}$ of $\left\{v_{j}\right\}$. Assume that $\left\{v_{j}^{\prime}\right\}$ converges to $v \in G u$. Take $I^{\prime}$ such that $i^{\prime} \leq j^{\prime}$ implies $v_{j} \preccurlyeq w_{j} \preccurlyeq w_{j}^{\prime}$. One writes

$$
\begin{aligned}
\gamma\left(d\left(v_{j}, v\right)\right) & =\lim _{j^{\prime}} \gamma\left(d\left(v_{j}, w_{j}^{\prime}\right)\right) \\
& \leq \lim _{j^{\prime}}\left(\phi\left(v_{j}\right)-\phi\left(w_{j}^{\prime}\right)\right)=\phi\left(v_{j}\right)-\phi(v) .
\end{aligned}
$$


So $v_{j} \preccurlyeq v$ for all $j$. Also,

$$
\begin{aligned}
\gamma(d(u, v)) & =\lim _{j} \gamma\left(d\left(v_{j}, v\right)\right) \leq \lim _{j}\left(\phi\left(v_{j}\right)-\phi(v)\right) \\
& =\phi(u)-\phi(v) .
\end{aligned}
$$

So $u \leqslant v$ and $u \in M$. Thus, $\left\{u_{j}\right\}$ has an upper bound in $M$.

By Zorn's Lemma, there is a maximum element $u^{*} \in M$. also, there is $v^{*} \in G u^{*}$ so that $u^{*} \preccurlyeq v^{*}$. Using (H1), there is $z^{*} \in G v^{*}$ so that $v^{*} \preccurlyeq z^{*}$. Hence, $v^{*} \in M$. The element $u^{*}$ is maximum in $M$, so $v^{*}=u^{*}$ and $u^{*} \in G u^{*}$. That is, $u^{*}$ is a fixed point of $G$.

Theorem 13. Let $(\mathscr{E}, d, \preccurlyeq)$ be a complete ordered metric space and $\phi: \mathscr{E} \longrightarrow(-\infty,+\infty)$ be a continuous function bounded below. Let $G: \mathscr{E} \longrightarrow \mathcal{N}(\mathscr{E})$ be a multivalued mapping. Assume that

(H1)For any $u \in \mathscr{E}$, there is $v \in G u$ so that $v \leqslant u$

(H2) $G(u)$ is compact for any $u \in \mathscr{E}$.

Then, G has a fixed point.

Remark 14. If $G$ is a continuous single-valued mapping in Theorem 6 (resp., Theorem 7), we can replace the condition (H) by

$$
\left(H^{\prime}\right): \text { for each } u \in \mathscr{E}, u \preccurlyeq G u(\text { resp., } G u \preccurlyeq u) \text {, }
$$

and we can obtain the same result.

Remark 15. If in Theorem 8 (resp., Theorem 10), $G$ is assumed to be a continuous single-valued mapping, then we get the same result when replacing the conditions (i) and (ii) by

(i) $G$ is monotone increasing (resp., decreasing), that is, for $u \preccurlyeq v$, we have $G u \preccurlyeq G v(\operatorname{resp}$., $G v \preccurlyeq G u)$

(ii) there exists $u_{0}$ with $u_{0} \preccurlyeq G u_{0}$ (resp., $G u_{0} \preccurlyeq u_{0}$ ).

If in addition $\phi$ is order upper (resp., lower) semicontinuous on $\mathscr{E}$, then $u^{*}$ is the smallest (resp., largest) fixed point of $G$ in $K=\left\{u \in \mathscr{E} \mid \operatorname{ss} u_{0} \preccurlyeq u\right\}$ (resp., $K=\left\{u \in \mathscr{E} \mid u \preccurlyeq u_{0}\right\}$ ).

Proof. Let $z$ be a fixed point of $G$ in $K$, i.e., $z=G z$. Since $u_{0} \preccurlyeq z$, we get $G u_{0} \preccurlyeq G z$. Hence, $u_{1}=G u_{0} \preccurlyeq G z=z$, i.e., $u_{1} \preccurlyeq z$. Suppose $u_{n} \preccurlyeq z$, then $G u_{n} \preccurlyeq G z$, i.e., $u_{n+1}=G u_{n} \preccurlyeq G z=z$. By a mathematical induction, we have $u_{n} \leqslant z$ for all $n$, then

$$
\begin{aligned}
\gamma\left(d\left(u^{*}, z\right)\right) & =\lim _{n \rightarrow \infty} d\left(u_{n}, z\right) \leq \varlimsup_{n \infty}\left(\phi\left(u_{n}\right)-\phi(z)\right) \\
& =\varlimsup_{n \infty} \phi\left(u_{n}\right)-\phi(z) \leq \phi\left(u^{*}\right)-\phi(z) .
\end{aligned}
$$

That is, $u^{*} \preccurlyeq z$.
Note that if we omit the continuity of $G$ and add above condition on $\phi$, the results remain true.

\section{Data Availability}

The data used to support the findings of this study are available from the corresponding author upon request.

\section{Conflicts of Interest}

The authors declare that they have no competing interests regarding the publication of this paper.

\section{Authors' Contributions}

All authors contributed equally and significantly in writing this article. All authors read and approved the final manuscript.

\section{References}

[1] J. Caristi, "Fixed point theorems for mappings satisfying inwardness conditions," Transactions of the American Mathematical Society, vol. 215, pp. 241-251, 1976.

[2] H. Aydi, E. Karapinar, and W. Shatanawi, "Coupled fixed point results for $(\psi, \varphi)$-weakly contractive condition in ordered partial metric spaces," Computers and Mathematics with Applications, vol. 62, no. 12, pp. 4449-4460, 2011.

[3] T. G. Bhaskar and V. Lakshmikantham, "Fixed point theorems in partially ordered metric spaces and applications," Nonlinear Analysis: Theory, Methods \& Applications, vol. 65, no. 7, pp. 1379-1393, 2006.

[4] D. Đorić, Z. Kadelburg, and S. Radenović, "Coupled fixed point results for mappings without mixed monotone property," Applied Mathematics Letters, vol. 25, no. 11, pp. 1803$1808,2012$.

[5] Z. Kadelburg, P. Kumam, S. Radenović, and W. Sintunavarat, "Common coupled fixed point theorems for Geraghty-type contraction mappings using monotone property," Fixed Point Theory and Applications, vol. 2015, no. 1, 2015.

[6] V. Lakshmikantham and L. Ćirić, "Coupled fixed point theorems for nonlinear contractions in partially ordered metric spaces," Nonlinear Analysis: Theory, Methods \& Applications, vol. 70, no. 2, pp. 4341-4349., 2009.

[7] V. Parvaneh, M. R. Haddadi, and H. Aydi, "On best proximity point results for some type of mappings," Journal of Function Spaces, vol. 2020, Article ID 6298138, 6 pages, 2020.

[8] S. Radenović, "Bhaskar-Lakshmikantham type-results for monotone mappings in partially ordered metric spaces," International Journal of Nonlinear Analysis and Applications, vol. 5, no. 2, pp. 37-49, 2014.

[9] S. Radenović, "Coupled fixed point theorems for monotone mappings in partially ordered metric spaces," Kragujevac Journal of Mathematics, vol. 38, no. 2, pp. 249-257, 2014.

[10] J. Vujaković, H. Aydi, S. Radenović, and A. Mukheimer, "Some remarks and new results in ordered partial b-metric spaces," Mathematics, vol. 7, no. 4, p. 334, 2019.

[11] X. Zhang, "Fixed point theorems of monotone mappings and coupled fixed point of mixed monotone mappings in ordered metric space," Acta Mathematica Sinica, vol. 44, no. 4, pp. 641-646, 2001. 
[12] X. Zhang, "Fixed point theorems of multivalued monotone mappings in ordered metric spaces," Applied Mathematics Letters, vol. 23, no. 3, pp. 235-240, 2010.

[13] R. P. Agarwal and M. A. Khamsi, "Extension of Caristi's fixed point theorem to vector valued metric spaces," Nonlinear Analysis: Theory, Methods \& Applications, vol. 74, no. 1, pp. 141-145, 2011.

[14] K. J. Chung, "Nonlinear contractions in abstract spaces," Kodai Mathematical Journal, vol. 4, no. 2, pp. 288-292, 1981.

[15] S. B. Nadler, "Multivalued contraction mappings," Pacific Journal of Mathematics, vol. 30, no. 2, pp. 475-488, 1969.

[16] N. A. Assad and W. A. Kirk, "Fixed point theorems for setvalued mappings of contractive type," Pacific Journal of Mathematics, vol. 43, no. 3, pp. 553-562, 1972.

[17] E. Ameer, H. Aydi, M. Arshad, and M. De la Sen, "Hybrid Ćirić type graphic $\Upsilon, \Lambda$-contraction mappings with applications to electric circuit and fractional differential equations," Symmetry, vol. 12, no. 3, p. 467, 2020.

[18] N. Alamgir, Q. Kiran, H. Isik, and H. Aydi, "Fixed point results via a Hausdorff controlled type metric," Advances in Difference Equations, vol. 2020, no. 1, 2020.

[19] H. Aydi, A. Felhi, E. Karapinar, and S. Sahmim, "A Nadler-type fixed point theorem in dislocated spaces and applications," Miskolc Mathematical Notes, vol. 19, no. 1, pp. 111-124, 2018.

[20] H. Aydi, M. Abbas, and C. Vetro, "Partial Hausdorff metric and Nadler's fixed point theorem on partial metric spaces," Topology and its Applications, vol. 159, no. 14, pp. 32343242, 2012.

[21] H. Qawaqneh, M. S. Noorani, W. Shatanawi, H. Aydi, and H. Alsamir, "Fixed point results for multi-valued contractions in b-metric spaces," Mathematics, vol. 7, no. 2, p. 132, 2019.

[22] N. Tahat, H. Aydi, E. Karapinar, and W. Shatanawi, "Common fixed points for single-valued and multi-valued maps satisfying a generalized contraction in G-metric spaces," Fixed Point Theory and Applications, vol. 2012, no. 1, 2012.

[23] Y. Q. Feng and S. Y. Liu, "Fixed point theorems for multivalued contractive mappings and multi-valued Caristi type mappings," Journal of Mathematical Analysis and Applications, vol. 317, no. 1, pp. 103-112, 2006.

[24] Y. Q. Feng and S. Y. Liu, "Fixed point theorems for multivalued increasing operators in partial ordered spaces," Soochow Journal of Mathematics, vol. 30, pp. 461-469, 2004.

[25] M. A. Khamsi, "Remarks on Caristi's fixed point theorem," Nonlinear Analysis: Theory, Methods \& Applications, vol. 71, pp. 227-231, 2009.

[26] Z. Li, "Remarks on Caristi's fixed point theorem and Kirk's problem," Nonlinear Analysis: Theory, Methods \& Applications, vol. 73, no. 12, pp. 3751-3755, 2010.

[27] Z. Li, "Fixed point theorems in partially ordered complete metric spaces," Mathematical and Computer Modelling, vol. 54, pp. 69-72, 2011. 